\title{
Biomarkers in COPD: the search continues!
}

\author{
David M. Mannino
}

Affiliation: Dept of Preventive Medicine and Environmental Health, University of Kentucky College of Public Health, Lexington, KY, USA.

Correspondence: David M. Mannino, Dept of Preventive Medicine and Environmental Health, University of Kentucky College of Public Health, 111 Washington Avenue, Lexington, KY 40536, USA.

E-mail: dmannino@uky.edu better COPD treatment http://ow.ly/HHPKl

In a working group of the National Institutes of Health focusing on biomarkers and surrogate end-points, a biomarker was defined as a "characteristic that is objectively measured and evaluated as an indicator of normal biologic processes, pathogenic processes, or pharmacologic responses to a therapeutic intervention" [1]. In the same document, the National Institutes of Health defined a clinical end-point as "a characteristic or variable that reflects how a patient feels, functions, or survives" and a surrogate end-point as a "biomarker that is intended to substitute for a clinical endpoint. A surrogate endpoint is expected to predict clinical benefit (or harm or lack of benefit or harm) based on epidemiologic, therapeutic, pathophysiologic, or other scientific evidence" [1].

Biomarkers are a critical component of the drug development and approval process. For chronic obstructive pulmonary disease (COPD) the only biomarker currently widely used in drug trials is lung function testing, typically forced expiratory volume in $1 \mathrm{~s}$ (FEV1). Although FEV1 is easy to obtain and reproducible it does not inform about underlying disease activity, does not separate out phenotypes of COPD, is not specific to COPD and is unresponsive to some therapies that clearly improve survival (such as long-term oxygen therapy) [2]. In 2009, Sin and Vеsтво [2] reviewed the current state of biomarkers in COPD and proposed the following criteria for developing novel biomarkers or other surrogate markers in COPD. 1) Is there a strong biological plausibility in terms of its role in the pathogenesis of disease? 2) Is there a strong, consistent and independent association between the biomarker and COPD? 3) Is there a strong, independent association between the biomarker and hard clinical outcomes such as mortality and hospitalisation? 4) Is there evidence from randomised controlled trials that the biomarker is modifiable by interventions? 5) Is there evidence from randomised controlled trials that changes in the biomarker status result in changes in an important (and accepted) clinical outcome (e.g. mortality, exacerbation, rate of decline in FEV1 and health status)?

In a recent review of COPD-related biomarkers, LocK-JOHANSSON et al. [3] examined recent data on surfactant protein $\mathrm{D}$, club cell protein 16 , pulmonary and activation-regulated chemokine/CCL-18, $\mathrm{C}$-reactive protein and fibrinogen. They concluded that none of these biomarkers fulfilled all of the criteria of Sin and Vestbo [2], particularly with regard to the final one, that evidence from a randomised clinical trial demonstrates that a change in the biomarker status results in a change in a clinical outcome. They also noted that either additional biomarkers, or perhaps combinations of biomarkers, might be needed to improve the sensitivity and specificity for the prognosis of COPD.

In this this issue of the European Respiratory Journal, LeUnG et al. [4] attempt to address, in part, this gap noted by Lock-Johansson et al. [3]. Leung et al. [4] demonstrate that higher levels of plasma pro-surfactant protein B (pro-SFTPB), in a cohort of current and former smokers at risk for developing lung cancer, was a modest predictor of lung function decline (refer to tables 3 and 4 in the article), accounting for a small

Received: Dec 262014 | Accepted after revision: Jan 062015

Conflict of interest: Disclosures can be found alongside the online version of this article at erj.ersjournals.com

Copyright CERS 2015 
proportion of the lung function decline at 1 and 2 years of follow-up. In addition, as is noted in table 2 in the article, the greatest effect in lung function decline was seen when going from the lowest quartile $\left(<16.93 \mathrm{ng} \cdot \mathrm{mL}^{-1}\right)$ to the top three quartiles $\left(\geqslant 16.94 \mathrm{ng} \cdot \mathrm{mL}^{-1}\right)$; there was no difference in lung function decline between the top three quartiles of this measurement. Finally, as is noted in table 4 in the article, the strongest predictor of plasma pro-SFTPB levels was current smoking status. A previous study by SIN et al. [5], in this same cohort, demonstrated that pro-SFTPB was a predictor of incident lung cancer, with similar results being found in data from the Carotene and Retinol Efficacy Trial (CARET) study.

The study by LEUNG et al. [4] nicely highlights some of the issues that emerge with biomarkers in COPD. First, most COPD research in the developed world has focused on current and former smokers. Growing literature has examined COPD in never-smokers, particularly in the developing world [6]. The factors that predict COPD development and progression in smokers may differ from those in never-smokers. Biomarkers may also be very different between never-smokers and current or former smokers. Secondly, many trials look at very short-term outcomes, given the long duration and variability in the natural history of COPD [7-9]. In the study by LeUNG et al. [4], the differences between the 1- and 2-year analyses are impressive. As COPD develops and progressives over many years (with a great deal of variability between individuals), studies with much longer durations are needed to fully assess the utility of this and other potential biomarkers. Finally, it is unlikely that this biomarker would be easily accessible to clinicians or clinical researchers in the future (although this could rapidly change if this biomarker becomes very useful in predicting outcomes or response to certain therapies).

In order to address problems in the development and approval of biomarkers for COPD, in 2010 the COPD Foundation established the COPD Biomarkers Qualification Consortium (CBQC). This is a partnership between the COPD Foundation, the pharmaceutical industry and the US Food and Drug Administration to pool industry and publically funded data to facilitate the development and approval of new therapies for COPD [10]. To date, one report has been submitted to the US Food and Drug Administration and the European Medicines Agency on fibrinogen [11]. This submission demonstrates that people with COPD and elevated fibrinogen levels are more likely to die (fig. 1) and have more exacerbations than people with COPD with normal fibrinogen levels. The hope is that this knowledge could be used to enhance study populations for clinical trials. Additional biomarkers are being evaluated, including measures of quality of life, measures of exercise capability and radiographic measures. As part of this process, additional biomarkers are being considered by the consortium as data becomes available.

Does pro-SFTPB have promise as a biomarker? Maybe. Its most promising feature is that it actually originates in the lung, which is not true for several other biomarkers that have been evaluated to date. Ultimately, it may become part of a panel of markers that allow us to better predict response to therapy and prognosis.

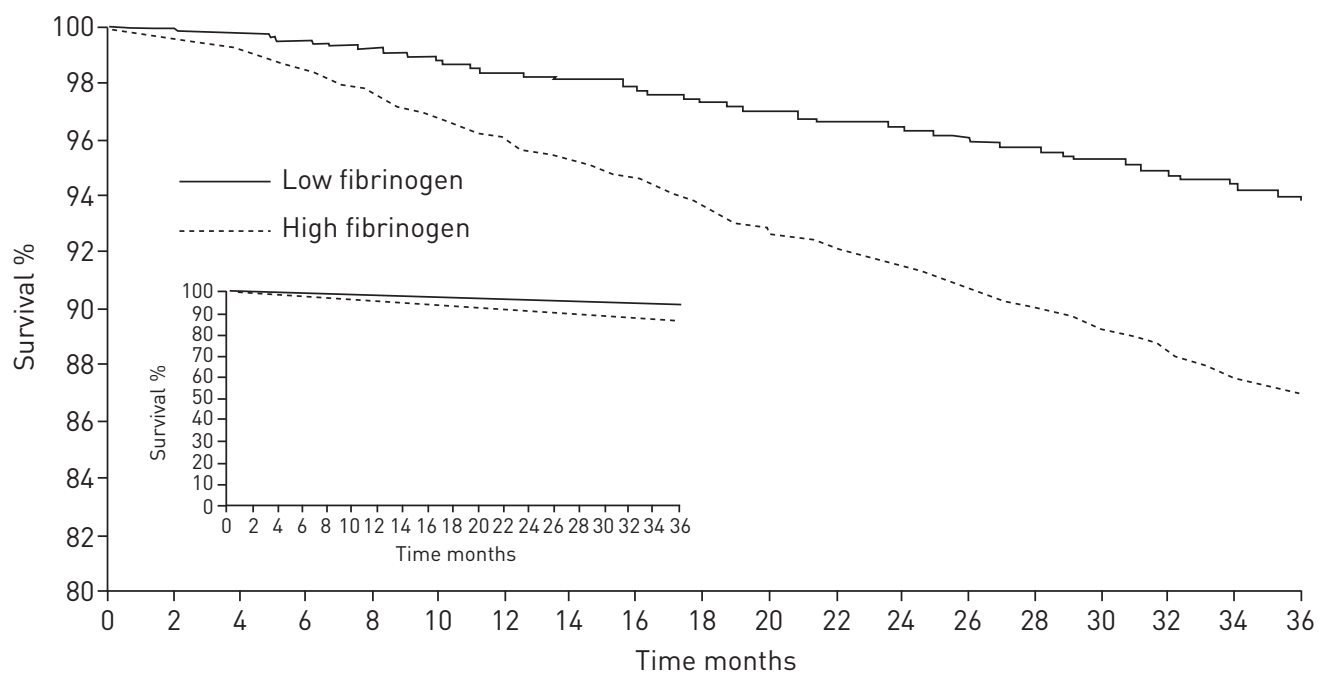

FIGURE 1 Survival of chronic obstructive pulmonary disease patients with high $\left(>350 \mathrm{mg} \cdot \mathrm{dL}^{-1}\right)$ versus low $\left(\leqslant 350 \mathrm{mg} \cdot \mathrm{dL}^{-1}\right.$ ) fibrinogen levels. Low fibrinogen: patients, $n=3523$; outcomes, $n=213(6 \%)$. High fibrinogen: patients, $\mathrm{n}=2853$; outcomes, $\mathrm{n}=372$ (13\%). Reproduced from [11] with permission from the publisher. 


\section{References}

1 Biomarkers Definitions Working Group. Biomarkers and surrogate endpoints: preferred definitions and conceptual framework. Clin Pharmacol Ther 2001; 69: 89-95.

2 Sin DD, Vestbo J. Biomarkers in chronic obstructive pulmonary disease. Proc Am Thorac Soc 2009; 6: 543-545.

3 Lock-Johansson S, Vestbo J, Sorensen G. Surfactant protein D, club cell protein 16, pulmonary and activation-regulated chemokine, C-reactive protein, and fibrinogen biomarker variation in chronic obstructive lung disease. Respir Res 2014; 15: 147.

4 Leung JM, Mayo J, Tan W, et al. Plasma pro-surfactant protein B and lung function decline in smokers. Eur Respir J 2015; 45: 1037-1045.

5 Sin DD, Tammemagi CM, Lam S, et al. Pro-surfactant protein B as a biomarker for lung cancer prediction. J Clin Oncol 2013; 31: 4536-4543.

6 Smith M, Li L, Augustyn M, et al. Prevalence and correlates of airflow obstruction in 317 000 never-smokers in China. Eur Respir J 2014; 44: 66-77.

7 Suzuki M, Makita H, Ito YM, et al. Clinical features and determinants of COPD exacerbation in the Hokkaido COPD cohort study. Eur Respir J 2014; 43: 1289-1297.

8 Miller A, Raskin JM. The natural history of COPD: confirming and going beyond Fletcher and Peto. Eur Respir J 2014; 44: 280-283.

9 Mannino DM, Watt G, Hole D, et al. The natural history of chronic obstructive pulmonary disease. Eur Respir J 2006; 27: 627-643.

10 Casaburi R, Celli B, Crapo J, et al. The COPD Biomarker Qualification Consortium (CBQC). COPD 2013; 10: 367-377.

11 Mannino DM, Tal-Singer R, Lomas DA, et al. Plasma fibrinogen as a biomarker for mortality and hospitalized exacerbations in people with COPD. J COPD F 2015; 2: 23-34. 